\title{
New Intelligent Technologies-Interactivity and Information Issues
}

\author{
Rusi Marinov \\ Department of Mass Communications, New Bulgarian University, Sofia, Bulgaria \\ Email: rmarinov@nbu.bg
}

How to cite this paper: Marinov, R. (2019). New Intelligent Technologies-Interactivity and Information Issues. Advances in Journalism and Communication, 7, 94-108. https://doi.org/10.4236/ajc.2019.73006

Received: June 14, 2019

Accepted: September 2, 2019

Published: September 5, 2019

Copyright ( 2019 by author(s) and Scientific Research Publishing Inc. This work is licensed under the Creative Commons Attribution International License (CC BY 4.0).

http://creativecommons.org/licenses/by/4.0/

\begin{abstract}
In this paper, attention is focused on the development of intelligent technologies in recent years, such as neural networks, deep learning, and artificial intelligence. In nowadays, interactive technologies have a major effect on the media and society. Mobile companies, social networks, various computer applications, smart devices constantly collect data for people without they know it and they are used to develop intelligent software systems to predict future behaviour. The article also describes some models of building an interactive space using new technologies, considering the trends in the development of information ecology. This research also analyses the following very important topics related to interactive technology, and intelligent systems: conversation in the interactive space, smart communications, big data and analytical techniques, mobile technology and some aspects of communication problems in the 21 st century.
\end{abstract}

\section{Keywords}

Interactive Technologies, Media Communications, Neural Networks, Artificial Intelligence, Smart Communications, Deep Learning, Information, Critical Issues

\section{Introduction}

The aim of this report is to demonstrate the importance of the new technology development, from one hand, and to show some threats for the citizens and media, from another hand. The problems of developing intelligent and smart technologies have two dimensions technical and non-technical. Technological problems affect the construction of the network, the introduction of communication protocols and the definition of mechanisms involved in intelligent objects. A special Internet Protocol (IP) for Smart Object Alliance (IPSO Alliance) has al- 
ready been created. Another problem is the standardization of smart sites or it is one of the most important factors in the development of technology. Intelligent systems are characterized by a huge number of devices, applications of many manufacturing companies with different specializations. With the help of standardization, on the part of manufacturers and system equipment, a new Internet system should be built. The more one technology is standardized, the greater of its successful application in practice and its acceptance by the business. In the past few years, there has been a boom in publications in the media, on websites and in specialized journals about the role and importance of artificial intelligence for economics and innovation. Projections of the development of such technologies are more than optimistic, although real achievements are not so noticeable. It can be said that there are more problems than significant success. Technology development ahead of the pace of building competencies in the web environment. Undoubtedly, there is a huge impact of new intelligent platforms on the life of people and the development of new media platforms. The balance of the media ecosystem in the modern world of information and network environment plays an important role in preventing the creation of sub-systems or conceptual orientations leading to mass control over the habits and behavior of citizens.

The French researcher of modern computer technologies (Lafon, 2004) explores human-computer interaction through the prism of interactivity. According to him, there are three main models of interactivity: a computer is considered as a means; computer as a partner; computer as a medium. We are interested in the latest model, in which computers serve to communicate with people through e-mail, chat rooms and video conferencing. The interactive model has three dimensions and features:

1) Descriptive: ability to identify impact and existing interfaces;

2) Evaluative: allows access to the development of various alternatives;

3) Generative power: helps designers refine and create a new design.

The evolution of media technologies over the past 200 years has focused on: newspapers; phone; moving pictures; radio; TV; cable TV; the Internet; web and semantic networks; network based on data; Big data; machine learning; media based on AI. Next, we look at the characteristics of the five basic technologies (Computer Business Review, 2018) that will influence on the media in the 21st century:

- Formation of personal networks using mobile devices;

- Design of special software that provides verbal communication through computers and launches video chats;

- Social networks are becoming a part of business and major news channel; social media as a means of communication and interaction, providing an exchange of opinions and ideas;

- Appearance of functional touch screens, virtual keyboards, the boom of smartphones, huge amount of mobile applications; 
- Development of cognitive machine as a Watson (IBM) with opportunities for interactive, compatible computer systems, machine learning, automatic answer to questions, evaluation and interpretation of huge information archives. Watson works using smart information architecture related to deep learning.

\section{Intelligent Technologies}

A special website (whatis.com, 2019) says that an intelligent system is a machine with embedded computers connected to the internet that can collect, analyse data and interact with other systems. The main features of such a system are the ability to learn from their own experience, to adapt to the situation in accordance with the data obtained, to manage remotely. Intellectual systems include not only "smart" devices, but also a set of other similar devices. A more sophisticated intelligent system is based on specialized software and may include chat and expert systems. In this case, embedded systems can process and analyse data or specialize in a specific type of task. An intelligent system can also be an important component of the Internet, providing automatic data transfer based on human-computer interaction. For example, technology-based intelligent data analysis, artificial intelligence, and neural networks were introduced in the 1960s, being the subject of many studies, publications, experiments, and developed projects. Interactive technologies and architectures, big data, neural networks have been actively developing in recent years as a result of improved capabilities of computer systems and accumulated knowledge in these areas of modern science. In this case, one can hardly speak of a "revolution", but rather there is a slow gradual accumulation of experience and knowledge transfer until the current level of network technologies is reached. It can be said that this is the Papert's Principle: "some of the most crucial steps in mental growth are based not simply on acquiring new skills, but on acquiring new administrative ways to use what one already knows".

Most of the research in the field of "artificial intelligence" is devoted to finding methods that can divide the problem into small sub-tasks, and then, if necessary, divide into even smaller parts. The most effective way to solve a problem is to know how to do it now. Under this condition, we can avoid the entire search process. Another area in the field of artificial intelligence is to find ways to integrate knowledge into machines. The problem here is how we discover the knowledge we need in order to learn how to represent this knowledge and, finally, develop a process that evaluates the effectiveness of our knowledge. In 1968, Marvin Minsky and Seymour A. Papert published the book "Perceptrons", demonstrating the capabilities and limitations of a simplified neural network. This book is also the first parallel study using a computer. Work is important for the further development of artificial intelligence. The idea that protects authors is that intelligence can arise from the activity of networks, such as neurons. Some elements of AI computers include the following basic actions: speech rec- 
ognition, self-study, planning and troubleshooting. Research in the field of artificial intelligence are high-tech and specialized. The main problem in this case is how to program computers to gain knowledge; logics; identify problems; planning ability; perception; possess properties for manipulating and moving objects. Knowledge engineering is an important part of AI/Artificial Intelligence/research. Machines must be able to act and react in the same way as humans, provided they have enough information about the world. It is assumed that artificial intelligence platforms have access to objects, categories, qualities, and corresponding relationships between these important elements and can design things. Machine learning is another important part of AI. Training without external control still requires the ability to identify patterns depending on the input information at the system input. On the other hand, machine perception is associated with the ability to use data from sensors integrated into the external environment; computer vision means the ability to analyse a visual signal.

In the specialized website, (techtarget, 2019) artificial intelligence is defined as a kind of simulation of human intelligence, implemented using machines and computer systems. The main processes in this case are training, obtaining information, arguing and correcting behaviour when conditions change. The practical application of AI can be implemented in the field of expert speech recognition and machine vision systems. The term "artificial intelligence" was commissioned in 1955 by American computer scientist John McCarthy, at a conference in Dartmouth, and has since developed as a separate discipline. In 1958, McCarthy created a special programming language called LISP. In the 1980s, during the boom in the development of artificial intelligence, the LISP language became the favourite of programmers who develop software to solve complex problems. This is the era of LISP machines manufactured by various companies, the most famous of which is Symbolics. Minsky, along with McCarthy in 1959, developed the "Artificial Intelligence Project" at the Massachusetts Institute of Technology. Today is called the "Laboratory of Computer Science of Artificial Intelligence." Minsky (Encyclopedia Britannica, 2019) defines AI as the science of creating machines that perform actions that require intelligence, if they are made by people. In 1986, he published his book, "The Society of mind". In the vocabulary (Minsky,1985) developed at the end of the books he defined the basic concepts related to the direction of intelligence and artificial intelligence, which are shown below to identify semantic dependencies.

Artificial intelligence-area of research solutions as machines to do things in a way that corresponds to intelligence.

Interaction-the effects that one part of the system has on the other. It should be noted that in the history of science, almost all phenomena are explained in terms of the interaction between parts during.

Another area in the field of artificial intelligence is to find ways to integrate knowledge into machines. The problem here is how we discover the knowledge we need in order to learn how to represent this knowledge and, finally, develop a 
process that evaluates the effectiveness of our knowledge. The learning (Minsky, 1985) has two sides. Some of our mind learns to memorize success when some methods work successfully. Other parts of our mind learn mainly when we make mistakes, recalling circumstances in which individual methods do not work properly. Therefore, learning to succeed focuses on how we think, while learning to fail leads to productive thinking, but there is a less direct approach to things. Naturally, people learn more than their success than failure. On the other hand, learning "positive" learning leads to minor improvements. We can assume that there is no way to avoid some discomfort when we make significant changes in our thinking. In the online version of the encyclopaedia, artificial intelligence is defining as the ability of digital computers or computer-controlled robots to perform tasks typical of humans. This term is often used to describe projects related to the development of systems with intellectual characteristics typical of people, such as discovery, logical approach, generalization and study from previous experience. Despite advances in computer science, no program has yet been created that would correspond to human intelligence and flexibility, especially for tasks requiring a broader view of things and daily updating of knowledge. On the other hand, some programs can successfully solve expert and specific tasks; in this sense, artificial intelligence has limited application in such areas as medical diagnostics, computers for information retrieval, speech and text recognition. The purpose of an intelligent system is to represent relevant cognitive functions using problem-solving techniques and machine learning technology. Specialized site on the new technology "fluid" analyses the achievements of IBM in the field of artificial intelligence to solve problems. Company teams use $\mathrm{AI}$ in three main areas: creating solutions for production problems; more effectively use people's skills; improved business timelines.

Examples of implementation AI technologies in business practice:

- Automate system processes/robots can be trained to perform repetitive or routine tasks, usually performed by humans;

- Machine and reinforcement learning;

- Machine vision-the science of computer vision, used in medicine to analyse various types of photographs, as well as to identify signatures;

- Management of processes related to the recognition of human languages (NLP). It is used in the recognition of spam messages, text translation, speech recognition, and analysis of feelings;

- Robotics-engineering sciences, focused on the design and manufacture of robotic systems.

\section{Interactivity and Interactive Communications}

The interactive communications are associated with new technologies and include the following basic elements: building an integrated information system; Intranet interactive panels and displays; Platform for big data applications for managing mobile devices; platforms for transferring knowledge and content; 
strategic communications team. The interactive media is a hybrid of a smart machine and human-computer interaction. The processes of interaction with the machine can be understood as liberal or restrictive activities with the possibility of greater freedom or control over the environment.

Interactivity (from a business point of view) means the search for information and data, coordination, monitoring and implementation of business operations in order to more efficiently exchange goods, services, and ideas. Interaction effects all economies, especially in technologically advanced countries. Interactivity in business plays a universal role. Individuals and organizations work together to find the most effective way to exchange, structure, manage and integrate activities relevant to the company and monitor their implementation. Some example of interactive events: dialog/communication; data collection; solving problems on the way of cooperation. In recent years we organized in the university several public workshops with the main subject related to interactive communications with more 100 participants. During the workshops, students present their own projects about interactive media and as a result, we defined some important principles about interactive communication from a practical point of view (https://bit.ly/2yLqAVk).

Our research show that the basic principles of interactivity should be:

○ variable content;

○ integrated internet technology;

○ platforms flexibility;

- scalar and modularity;

○ program code;

○ automation (in data processing and solutions);

$\circ$ an active participant in individual sessions;

- information and video in the form of streaming/streaming technology/;

○ mobile applications;

$\bigcirc$ availability from anywhere in the world;

- media as a translator of reality/recognition of images, objects, and voices, the conversion of speech into text and vice versa/;

o construction of augmented, mixed reality and extended reality/AR, MR, XR/;

- communication depends on the speed of information exchange

The future of interactive technologies will evolve in several key areas (See the Figure 1), fixed in the 4I model (fast company, 2012)

- The appearance of an immersive medium: searching for plot depth, using additional context and sensory experiment.

- Improved interactivity: allows the reader to become part of the story and increase the ability to influence the version of the story and the result.

- Integration: the presence of multidirectional connections between platforms and exceeding the limit for easy copying of content to devices.

- Impact: encourage consumers to take certain actions-buy products; subscription to services.

- Support for the cause 
Basic interactive model

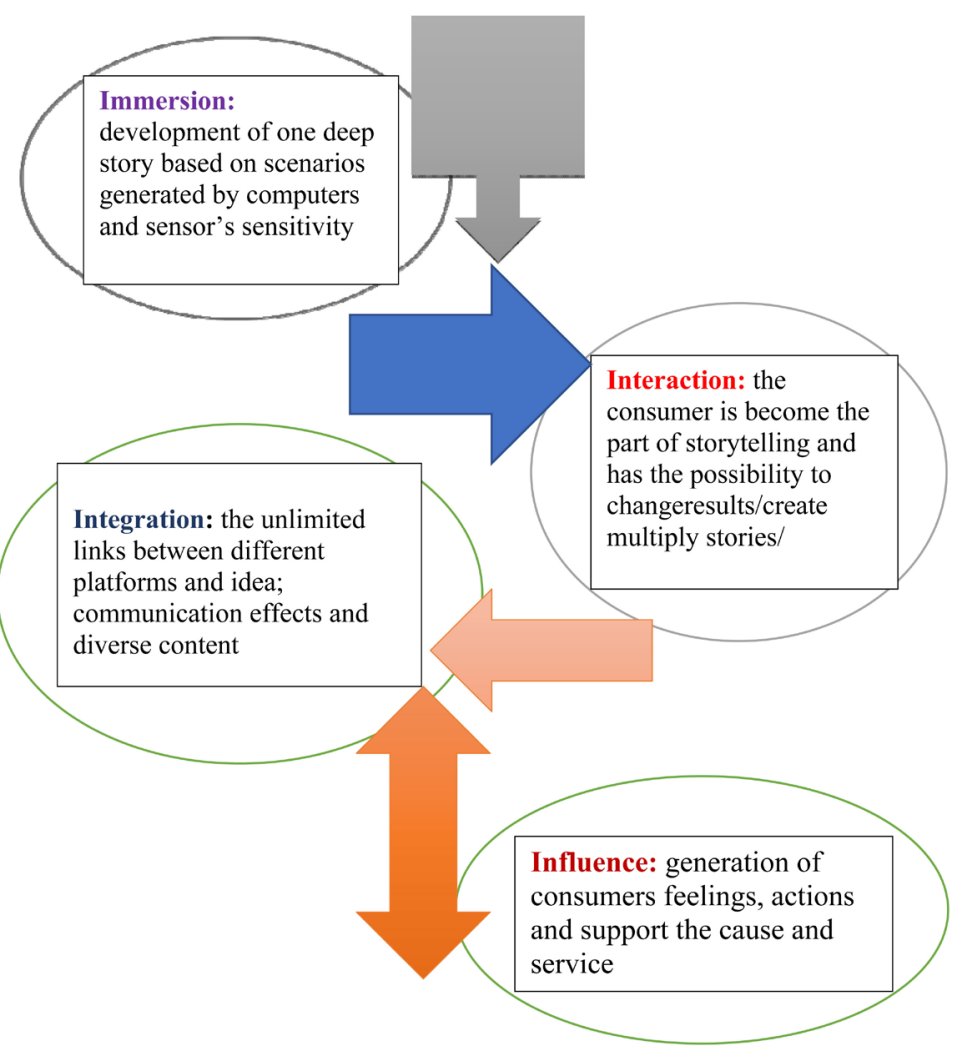

Figure 1. Structure of interactive communications.

From the media development point of view of related to communication technologies, several major transformations appear: the emergence of post-PC architectures; open-ended mass cooperation; integrated virtualization; collective intelligence; radical transparency; biometric identification; visualization of an open data type; possession of personalized personal information; the emergence of expert search engines that record online reputation; IT as a business unit; generate semantically related data. Interactive media, by definition, is digital and includes a combination of electronic texts, graphics, dynamic images and sounds structured in a computer environment, which allows people to interact with data to achieve specific goals. The digital environment, according to experts, includes the Internet, telecommunications, interactive, digital television. Digital space is characterized by a huge variety of information channels and data delivery platforms. With the advent of computer media or the so-called "digital", the term "new media" has appeared. In this case, the definition of "new" is added to the basic term "medium", which causes problems with the definitions. The term "interactive media" is considered more appropriate, with emphasis on interactivity as a key element or, in this case, the search for something significant that distinguishes traditional media from more modern ones. The confusion is com- 
pounded by the emergence of terms such as "social networks," primarily the success and boom of social networking sites such as facebook, myspace, and twitter. On these sites, open-source interactivity is inherent, but to a certain extent.

Interactivity (Rafaeli, 1997) is a process associated with a change in the characteristics of a communication medium. Like face-to-face communication, computer-mediated communication can provide a high level of interactivity. The result of these processes is the motivation and involvement of participants in the action. The interactivity characteristic of groups in a computer environment is best explained using interactivity. Interactivity is not a feature of the environment. Rather, these are processes that shape communication. It also determines how each subsequent message corresponds to a previous one or, more precisely, how later messages relate to earlier ones. Given the theories of Hoffmann (1967 and 1981), Bretz (1983), Rogers (1986), Tannen (1989), Zheglov (1992), Walter (1992). We can say that communication relates mainly to the goals of interaction. Interactivity is still the place to exchange interpreted context. Interactivity involves different ways of interacting with participants. Collective meaning is achieved through "conversation", which is seen as a repeating process of communication. Interactivity encompasses those forms of communication that are more personal, personalized and dialogue oriented. It is more logical to say that interactivity is an important model used to map the activities of groups using computer communication technologies, it is also defined as a hybrid design. Interactivity is the link between personal and mass communication, mediated by the media, and direct communication, between written and oral communication.

According to the classic Rafaeli (Rafaeli \& Sudwewks, 2006) design for interactivity, we can talk about three forms of communication:

1) Declarative or single level (typical for radio and television);

2) Reactive or two-stage advanced models;

3) Interactive-requires that subsequent messages must be compatible with previous ones but considering the response to them.

Jens Janson (Jensen, 1998) cites several forms of interactivity, considering the work of Raphael and other authors before him.

- Interactive depend of transmission-Measures media potential, providing a continuous flow of information, including user video, teletext, multi-channel systems, data transmission;

- Advisory interactivity-refers to two-level multimedia systems with a return channel request/online information services, electronic encyclopaedias, FTP, Gopher/;

- Conversation-oriented interactivity-allows the viewer to produce and enter their own information into media systems based on two-way communication and real-time video: examples here are news groups, video-conferencing systems and email/; 
- Registered interactivity-registration of information from the reader/viewer and adaptation of the media to the needs and actions of a person. The system is based on automatic response and adaptation. Examples: CCTV and response systems, smart agents, smart guides, smart interface.

Basic elements of the interactivity: media environment; active participation; to take initiatives: client-user relationships; biometric systems; autonomous work; technology transfer; assessment systems; and pedagogical approach. Our content analysis of websites of mainstream European media shows that used only 3 - 4 interactive element in order to communicate with potential users and consumers.

In the last $3-4$ years, the so-called interactive agents have also appeared (McGinley, 2019). In this case, studies from several scientific fields are combined, such as linguistics, computer graphics, virtual reality. They are usually managed using natural language, including teams, questions, and declarative sentences. Information exchange is a two-way process between a person and an object. This type of technology is also a kind of software and virtual objects that have environmental knowledge. Teams are focused on basic actions and behaviour in a real environment. Questions are focused on obtaining information or require some action. Declarative sentences provide information about agents, change their knowledge of the environment and teach them basic behaviour. The main components of the interactivity of this machine are sensors, controllers, knowledge, actuators. Controllers create appropriate behaviour based on agent knowledge.

The interaction takes place using a speech recognition system and includes physical actions and the study of new information. The platform for implementing this type of object is DLP. Interactivity is still relevant for networked smart devices that interact with almost all machines, from household appliances, telephones, cars, and to semantic web technologies, i.e. they are browser-specific, and programs can be customized, interacted, collaborated and complemented.

A popular example of such "agents" is VITA-Virtual Interactive Training Agent (Institute for creative technologies, 2014) it is a special virtual reality system focused on the formation of competencies during interviews for hiring. The program is designed to educate people with autism and other similar illnesses in order to build confidence in interviews. Continuing education is offered to successfully complete the interview in a simulated, secure virtual environment. The goal is to develop and improve the skills of people with a certain degree of disability. The project was developed by the Institute of Creative Technologies and is fully socially oriented.

The virtual chat site defines the characteristics of virtual "agents". In short, these are computer, animated, intelligent virtual "characters" that are used as online assistants. They provide conversations with users, answer questions and present adequate non-verbal behaviour in each situation. In the professional community, they are often called intelligent or interactive "agents." An example of such virtual software is Louise, eBay. 
In recent years, Microsoft has developed and sold special augmented reality glasses with interactive elements. Microsoft HoloLens (microsoft.com) achieves the so-called "mixed reality" or "mixed reality". MR-connects simultaneously on one platform: people, objects and places taken from the physical and digital world. In this case, we are talking about interaction based on a hologram, which allows you to visualize scenes and processes with digital content as part of the real world. Holograms respond to movements and gestures of both the participant and signals from the external environment. The platform allows you to interact with content and information in a natural way.

According to the Gartner Institute (gartner.com, 2016), by 2020 the share of autonomous software agents without human control used in economic transactions will be 5 percent. It may be noted that such structures are present now, but they are controlled by people. New autonomous software blocks will be used in corporations, in the legal system and in business. Gradually, he will move to a programmed, algorithmic economy, which will also require new models of financial services. In the future, there will be new opportunities for banking, currency exchange, insurance and market changes. Currently, mobile companies, social networks and various computer applications are constantly collecting data for people, unaware of this, and are further used to develop intelligent software systems to predict future behaviour. One of the ways to protect citizens is to change the approach to the use of technology. Currently, people do not understand the dark side of the platforms until they are involved in actions that lead to personal and financial losses. We, as consumers, have more opportunities to control the Internet and influence lifestyle. Today, platforms are limited to several technologies, such as Amazon Alexa, Google Home, Apple Siri, but very soon the data will attract us, such as television systems, cars, refrigerators, toys, drones, and so on. As a result, the collected personal data can be used in intelligent systems and algorithms that can manipulate the attention and behaviour of the consumer in order to make a profit for the owners of web platforms. Now we have control over the extent of the Internet impact of items, because they are very poorly distributed as technologies. Experts advise people to think about the negative aspects of technology right now, before it is too late. Our voice is our experience is paramount. Otherwise, anti-utopian technologies will control our life soon. Excessive reliance on Internet platforms as something good and positive causes people to ignore warning signals of danger.

From European perspective (ec.europa.eu, 2019) development of interactive technologies provokes design of the new generation of internet devices. Interactive technologies are at the center of developing a more human centric internet and are highly important for the cultural and creative industries. Technologies such as augmented and virtual reality are going to transform the ways in which people interact and share information on the internet and beyond. This will impact European industries ranging from the cultural to manufacturing, healthcare to education and open many new business opportunities. To benefit from the technological advances and the changes they bring to society, internet users will 
need improved information access and more natural ways to use these new technologies. Speech and vision perception, wearable technologies, haptics, audio and video rendering and even brain-computer interfaces will be used to develop new interaction systems and improve user experience.

\section{Information Problems and Issues}

In the report from 2019, in the "Technology Review" (Giles, 2019) is analysed five threats related to the development of new intelligent machines, which will be discussed in more detail below.

- Machines using AI can generate fake video and audio recordings. People can hardly tell how they correspond to what is happening. It is possible, with the help of false video from top managers of real companies, to provoke financial crises, especially when it is assumed that the video of top executives has economic problems. There are also "start-up projects" that offer software to detect fake video files, but their effectiveness and practical applicability are questionable

- Loss of protection against AI threats: security companies are trying to use artificial intelligence models to improve protection, but complex hacker attacks try to spoil such approaches. On the one hand, AI can facilitate the process of recognizing true noise signals, but on the other hand, such technologies in the hands of opponents can complicate attacks against business security.

- Hacking smart contracts. Intellectual contracts and related programs are stored in the form of blockchain stricture, which, of course, can automatically change the form of some digital assets depending on conditions. Such contracts and contracts are widely used in business for both the transfer of funds and the transfer of intellectual property rights and property. The main problem of blockchain technologies is that the design is designed and focused on full transparency. With this approach, the protection of data associated with smart contracts is a serious problem, and some start-up projects are oriented in this direction, offering software to protect such systems.

- Crack the code using the advantages of quantum computers. Soon, quantum computing systems will have enormous computational capabilities in terms of fast data and information processing. This will unimaginably violate the traditional network and computer codes. Quantum machines are in the process of being developed and improved, currently with limited capabilities, and a similar threat will arise in a few years. Recent reports and recommendations of professional expert groups on quantum electronic systems recommend switching to new models for programming and coding information in order to be resistant to quantum computer risks.

- Attacks from the computer cloud. A large business that stores data from other small companies on its servers or in computer centers is always a super task for professional hackers. Companies with cloud computing resources, 
such as Amazon, Google and Microsoft, are investing huge cybersecurity resources in their computer centres. The recommendations of the expert centres are to prepare enterprises in advance for such risks in the future in order to more effectively protect them from new technological threats.

The neural networks imitate human behaviour and the nervous system in a very specific way, and such networks are built up of several interconnected "nodes" that process information. Like neurons, these specific computer points receive information signals from neighbouring nodes, and then imitate their own signals. Neural networks are trained to solve computer problems by analysing vast amounts of data, and now they are becoming an indispensable part of artificial intelligent systems, speech recognition platforms, automatic translators and self-guided vehicles. Neural networks, on the other hand, are also "black box" systems, when they are trained once (considering that developers have ideas about the data and the processes that they will process), their behaviour can also be unpredictable In 2015 The Laboratory of computer science and Artificial intelligence at MIT has developed a special method for analysing processes in the so-called "black box" of a neural network trained to recognize visual scenes. Scientists present a fully automated version of the system. Today, neural networks are organized in the form of layers, and the data from the lower layers after processing are transmitted over the network.

Another publication (Wolchover, 2019) on the professional web site dedicated to new wired technologies discusses the problem area in the neural network known as the black box. Like the brain structure, deep neural networks have several neural layers, some of which are artificial fictions as part of computer memory. When neurons are activated, they send signals to the appropriate neurons from the upper levels, in-depth training, network connections are amplified or weakened, depending on the need to make the system more efficient when receiving input signals. The neural network can then learn from thousands of photographs or images stored in the system that help identify the object with high accuracy. Of special cases, a magical leap to generalized concepts occurs, and it transmits to the network additional power, like logical thinking typical of a person, in this case it can even be said about the creative process and other similar functions inherent in the intellect. Such structures are trained using a procedure known as "information transfer". The method can be used to analyse the learning process and for larger neural networks. In practice, experts are faced with a huge amount of information, and the problem is how to process the data so that the extracted signals correspond to the tasks performed by the network. From the theory of Shannon it is known that information has no relation to semantics, but Naftali Tishby believes that this is not so with the neural network. According to him, using information theory, the relevant information can be determined with great accuracy. In 2015, Nuga Slazovsky hypothesizes that deep learning is based on the principle of "transmitting information," which in practice means compressing the data that introduces 
noise into the system in order to preserve and preserve valuable information for the task being performed.

On the site of european TV "euronews" in the recent years has about 307 publications, but many of them are very naive from professional point of view. For example they pointed out about seven important principles (euronews.com, 2018) (this is only European conception).

- Accuracy-knowing what happens to business algorithms and the implications for companies;

- Transparency-to be open to change, to publicly talk about your problems and requires seven reliability, consistency, realism;

- Strategic approach-based on scenarios, presentation of the results of the strategy implementation/being part of the community-important requirements/;

- Collaboration-to help decision makers and policy makers explain massive changes.

We will also focus on some aspects of the impact of new technologies (facetofacedigital.com, 2018) on the media:

Accessibility: the technology allows you to transfer news and quality content using mobile applications and smartphones, the reader can reach the material much faster than with traditional means.

Use of social networks: today most of the media use active social networks such as facebook, twitter and instagram.

Involvement: through social networks, traditional news sites seek feedback and connect the reader with content. Comments help to improve the service of people in the future and get more information about interesting materials;

Podcasting: the perfect way to stream audio programs, for example, using phones and computers. People listening to podcasts are easily trained to pay attention to discussions about politics, national issues and finances.

- Streaming: a new wave-puts the person at the centre of what is happening. This is achieved through funds such as YouTube Live or Facebook Live.

Key areas for improving information domain:

- Mobility and ease transfer of media content;

- Stability-requirements as result of non-standard environment in difficult problem situations;

- Flexibility/interchangeability - creating unified and hybrid web platforms;

- Simplicity-ease of technology application and software, minimal costs for employee training and quick development;

- Accessibility-low costs when using technology.

\section{Conclusion}

The development of new technologies such as artificial intelligence, smart machines, deep learning, the formation of cyber-physical reality, automatic processing of huge data, interactive agents, the introduction of analytical and cloud 
resources, on the one hand, leads to accelerated development of business, media, and society, but, on the other hand, there are new issues, risks to the people, business and society that will require new types of professional skills and accelerated investments and innovations to protect the internet and network resources. Undoubtedly it is the fact of the impact of new intellectual platforms on the way people live and, on the development, and improvement of the media. Technological developments are ahead of the pace of building competencies in the web environment. In today's world, information literacy and network competence play an important role in the creation of one humanized media environment.

\section{Conflicts of Interest}

The author declares no conflicts of interest regarding the publication of this paper.

\section{References}

Beaudouin-Lafon, M. (2014). Interaction Design, Not Interfaces. https://dl.acm.org/citation.cfm?id=989865

Computer Business Review (2018). Top 5 Technological Advances of the 21st Century. https://www.cbronline.com/list/top-5-technological-advances-21st-century

Ec.europa.eu (2019). Next Generation Internet-Interactive Technologies. https://ec.europa.eu/digital-single-market/en/next-generation-internet-interactive-tech nologies

Encyclopedia Britannica (2019). https://www.britannica.com/biography/Marvin-Lee-Minsky

Euronews.com (2018). https://bit.ly/2TEX7W3

Facetofacedigital.com (2018). The Impact of New Technologies on the Media. http://www.facetofacedigital.com/the-impact-of-new-technology-on-mass-media/

Fast Company (2012). The Future of Interactivity and Storytelling. https://www.fastcompany.com/2681470/the-future-of-storytelling-immersion-integrati on-interactivity-impact

Gartner (2016). Predictions. http://www.gartner.com/newsroom/id/3143718

Giles, M. (2019). Five Emerging Cyber-Threats to Worry about in 2019. https://www.technologyreview.com/s/612713/five-emerging-cyber-threats-2019

Institute for Creative Technologies (2014). http://ict.usc.edu/prototypes/vita/

Jensen, J. F. (1998). Interactivity: Tracking a New Concept in Media and Communication Studies. Nordicom Review, 19, 185-204. http://bit.ly/2wy6rj8

McGinley, R. (2019). The Earth's Original 4.5-Billion-Year-Old Electronic Music Composition. http://www.interactive-agents.com/paper1.html http://www.zdnet.com/blog/collaboration/ubiquitous-intelligent-internet-agents/1294

Microsoft.com. HoloLens. https://goo.gl/KJQgh9 https://www.microsoft.com/en-us/hololens/why-hololens

Minsky, M. (1985). The Society of Mind (pp. 96, 102). New York: Simon \& Schuster.

Rafaeli, S., \& Sudweeks, F. (2006). Networked Interactivity. Journal of Computer-Mediated Communication, 2, JCMC243. https://doi.org/10.1111/j.1083-6101.1997.tb00201.x 
http://onlinelibrary.wiley.com/doi/10.1111/j.1083-6101.1997.tb00201.x/full\#f1

Techtarget (2019). http://searchcio.techtarget.com/definition/AI/

Whatis.com (2019). https://whatis.techtarget.com/search/query? q=intelligent+system

Wolchover, N. (2019). New Theory Cracks Open the Black Box of Neural Networks. https://www.wired.com/story/new-theory-deep-learning 